\title{
THE
}

\section{Signatures of quantum integrability and nonintegrability in the spectral properties of finite Hamiltonian matrices}

Vyacheslav V. Stepanov

Gerhard Müller

University of Rhode Island, gmuller@uri.edu

Follow this and additional works at: https://digitalcommons.uri.edu/phys_facpubs

Terms of Use

All rights reserved under copyright.

\section{Citation/Publisher Attribution}

Stepanov, V. V., \& Müller, G. (2000). Signatures of quantum integrability and nonintegrability in the spectral properties of finite Hamiltonian matrices. Physical Review E, 62(2), 2008-2017. doi: 10.1103/

PhysRevE.62.2008

Available at: http://dx.doi.org/10.1103/PhysRevE.62.2008

This Article is brought to you for free and open access by the Physics at DigitalCommons@URI. It has been accepted for inclusion in Physics Faculty Publications by an authorized administrator of DigitalCommons@URI. For more information, please contact digitalcommons-group@uri.edu. 


\title{
Signatures of quantum integrability and nonintegrability in the spectral properties of finite Hamiltonian matrices
}

\author{
Vyacheslav V. Stepanov and Gerhard Müller \\ Department of Physics, University of Rhode Island, Kingston, Rhode Island 02881-0817
}

(Received 2 March 2000)

\begin{abstract}
For a two-spin model which is (classically) integrable on a five-dimensional hypersurface in six-dimensional parameter space and for which level degeneracies occur exclusively (with one known exception) on fourdimensional manifolds embedded in the integrability hypersurface, we investigate the relations between symmetry, integrability, and the assignment of quantum numbers to eigenstates. We calculate quantum invariants in the form of expectation values for selected operators and monitor their dependence on the Hamiltonian parameters along loops within, without, and across the integrability hypersurface in parameter space. We find clear-cut signatures of integrability and nonintegrability in the observed traces of quantum invariants evaluated in finite-dimensional invariant Hilbert subspaces. The results support the notion that quantum integrability depends on the existence of action operators as constituent elements of the Hamiltonian.

PACS number(s): 05.45.-a, 75.10.Hk, 75.10.Jm
\end{abstract}

\section{INTRODUCTION}

An autonomous classical Hamiltonian system with two degrees of freedom, specified by some analytic function $H\left(p_{1}, q_{1} ; p_{2}, q_{2}\right)$ of canonical coordinates, is either integrable or nonintegrable-tertium non datur. If a second integral of the motion can be found, i.e., an analytic function $I\left(p_{1}, q_{1} ; p_{2}, q_{2}\right)$ which is functionally independent of $H$ and satisfies $d I / d t=\{H, I\}=0$, the system is proven integrable. If chaotic trajectories can be detected in the phase flow, the system is demonstrably nonintegrable. Although it may happen that neither evidence can be ascertained in practice for a given $H$, one or the other status is guaranteed to apply.

A question of long-standing interest has been whether an equally clear-cut classification of systems exists in quantum mechanics. Translating the criterion of classical integrability into quantum mechanics for systems with few degrees of freedom opens up loopholes of ambiguity that are not easily closed $[1,2]$. Quantum mechanically, a second integral of the motion, i.e., an operator $I$ with $[H, I]=0$ can always be constructed, for example, via time average of an arbitrary operator $A[3,4]$. Performing the time average in the energy representation eliminates all off-diagonal matrix elements of $A$. Which attributes of quantum invariants are most sensitive to the integrability status of the system?

Quantum chaos research has identified a catalog of attributes that distinguish quantized nonintegrable from quantized integrable systems [5-7]. The most widely studied distinctive properties pertain to level statistics. However, in the extreme quantum limit of a typical model system, where the density of energy levels is low, this distinction is blurry at best or altogether unrecognizable. Only in the energy range where the level density is high, which includes the semiclassical regime, do the contrasting level spacing distributions come into focus. Other indicators of quantum chaos are similarly ambiguous.

One unequivocal discriminant between quantized integrable and nonintegrable systems was recently identified in a study of level crossing manifolds in the parameter space of a two-spin model [8]. The system is specified by the quadratic Hamiltonian

$$
H=\sum_{\alpha=x, y, z}\left\{-J_{\alpha} S_{1}^{\alpha} S_{2}^{\alpha}+\frac{1}{2} A_{\alpha}\left[\left(S_{1}^{\alpha}\right)^{2}+\left(S_{2}^{\alpha}\right)^{2}\right]\right\}
$$

for two quantum spins $\mathbf{S}_{1}, \mathbf{S}_{2}$ of equal length $\sqrt{\sigma(\sigma+1)}(\sigma$ $\left.=\frac{1}{2}, 1, \frac{3}{2}, \ldots\right)$. In the classical limit $\hbar \rightarrow 0, \sigma \rightarrow \infty, \hbar \sqrt{\sigma(\sigma+1)}$ $=s$, the operators $\mathbf{S}_{i}$ turn into 3-component vectors, $\mathbf{S}_{i}$ $=s\left(\sin \vartheta_{i} \cos \varphi_{i}, \sin \vartheta_{i} \sin \varphi_{i}, \cos \vartheta_{i}\right)$, and Eq. (1) then describes the energy function of an autonomous Hamiltonian system with two degrees of freedom and canonical coordinates $p_{i}=s \cos \vartheta_{i}, q_{i}=\varphi_{i}, i=1,2$. The classical integrability condition was shown to have the form [9]

$$
\left(A_{x}-A_{y}\right)\left(A_{y}-A_{z}\right)\left(A_{z}-A_{x}\right)+\sum_{\alpha \beta \gamma=\operatorname{cycl}(x y z)} J_{\alpha}^{2}\left(A_{\beta}-A_{\gamma}\right)=0 .
$$

Quantum mechanically, the Hamiltonian (1) is expressible as a real symmetric block-diagonal matrix, where each of the infinitely many finite-dimensional blocks is associated with one spin- $\sigma$ realization of an irreducible representation of the underlying (discrete) symmetry group (see the Appendix).

The main conclusions of the level crossing study for this system may be summarized as follows [8]: (i) In the sixdimensional (6D) parameter space of (1), level degeneracies occur on smooth 4D structures [10] (ii) For an invariant block of $H$ with $K$ levels, this 4D structure consists of $K$ -1 sheets, each representing one twofold $[k, k+1]$ level degeneracy in the sequence $E_{1} \leqslant E_{2} \leqslant \cdots \leqslant E_{K}$. (iii) All 4D level crossing sheets are completely embedded in the $5 \mathrm{D}$ integrability hypersurface. (iv) Under mild assumptions, the integrability condition (2) can be determined analytically from the conditions of level degeneracy in low-dimensional invariant Hilbert subspaces of $H$.

These results strongly suggest that the notion of integrability remains meaningful for quantum systems described by 
finite Hamiltonian matrices, notwithstanding the fact that there exist universal algorithms for the diagonalization of finite symmetric matrices.

For a deeper understanding of this subtle notion of quantum integrability, we note that classical integrability guarantees the existence of a canonical transformation $\left(p_{1}, q_{1} ; p_{2}, q_{2}\right) \rightarrow\left(\mathcal{J}_{1}, \theta_{1} ; \mathcal{J}_{2}, \theta_{2}\right)$ to action-angle coordinates. It transforms the Hamiltonian $H\left(p_{1}, q_{1} ; p_{2}, q_{2}\right)$ and the second integral of the motion $I\left(p_{1}, q_{1} ; p_{2}, q_{2}\right)$ into analytic functions $H_{C}\left(\mathcal{J}_{1}, \mathcal{J}_{2}\right), I_{C}\left(\mathcal{J}_{1}, \mathcal{J}_{2}\right)$. Each point $\left(\mathcal{J}_{1}, \mathcal{J}_{2}\right)$ on the action plane specifies a torus in phase space. In the nonintegrable case, the actions $\mathcal{J}_{1}, \mathcal{J}_{2}$ are only defined for the surviving tori. Since the tori are no longer dense anywhere in phase space, no smooth functions $H_{C}, I_{C}$ on $\mathcal{J}_{1}, \mathcal{J}_{2}$ exist anymore.

In a companion paper [11] we have postulated that the underlying cause for the embedment of $\left(d_{I}-1\right)$-dimensional level crossing manifolds in a $d_{I}$-dimensional (classical) integrability manifold of the parameter space (with dimensionality $d \geqslant d_{I}$ ) is linked to the existence of action operators as constituent elements of the Hamiltonian. In that study we have demonstrated for two distinct model systems the explicit functional dependence $H_{Q}\left(\mathcal{J}_{1}, \mathcal{J}_{2}\right), I_{Q}\left(\mathcal{J}_{1}, \mathcal{J}_{2}\right)$ of the Hamiltonian and the second integral of the motion on two action operators, and compared it to the similar yet different functional dependence $H_{C}\left(\mathcal{J}_{1}, \mathcal{J}_{2}\right), I_{C}\left(\mathcal{J}_{1}, \mathcal{J}_{2}\right)$ of the corresponding classical invariants on the classical action coordinates.

The familiar controversies surrounding the notion of quantum action do not arise unless we insist on interpreting the action operator as a (stationary) canonical momentum, which calls for an angle operator. The main problem is how to define the latter in a satisfactory way $[12-14]$. If the action operator is defined solely on the basis of its spectral properties, there is no need for introducing angle operators.

\section{METHOD}

A more indirect but no less compelling method for demonstrating the existence of action operators as constituent elements of the quantum invariants $H, I$ in some regions of parameter space, namely on the integrability hypersurface, and their nonexistence elsewhere is pursued here for the twospin model (1). We investigate the functional dependence of the eigenvalues of quantum invariants on the Hamiltonian parameters, in particular across lines demarcating changes in symmetry and/or integrability status.

Here and in the following, all references to integrability status rely on the well understood and rigorously established classical integrability condition (2), but the focus is on the study of quantum mechanical properties that are sensitive to this condition and thus impart meaning to it as a quantum integrability condition.

On the integrability hypersurface (2), the natural quantum numbers of the eigenstates within any invariant Hilbert subspace of $H$ are the integer pairs $\left(m_{1}, m_{2}\right)$ specifying the eigenvalues (in units of $\hbar$ ) of the action operators $\mathcal{J}_{1}, \mathcal{J}_{2}$. Henceforth we call them action quantum numbers. Elsewhere in parameter space, where level crossings between eigenstates of the same parameter space are prohibited, the natural quantum number is a single integer, the energy sort- ing quantum number $n$. What consequences do these conflicting assignments of quantum numbers in the two regions of parameter space have for the functional dependence of quantum invariants on the Hamiltonian parameters?

Consider the case of a $K$-dimensional invariant subspace of (1) spanned by the basis given in the Appendix. The $K$ eigenstates $|k\rangle, k=1, \ldots, K$ then form a star of orthonormal vectors pointing in oblique directions with respect to the coordinate axes. A tiny change of the parameters $J_{\alpha}, A_{\alpha}$ causes the star of eigenvectors to rotate slightly. By monitoring the inner product between eigenvectors before and after every infinitesimal parameter change, we can keep track of all eigenvectors along the entire loop in parameter space.

At the same time, we monitor the effect of the gradually transforming eigenvectors on the eigenvalues of two quantum invariants. For this purpose we choose the energy expectation value $E_{k}=\langle k|H| k\rangle$ and the expectation value $I_{k}$ $=\langle k|A| k\rangle$, where $A$ is some function of the $S_{i}^{\alpha}$ [15]. When the Hamiltonian parameters $J_{\alpha}, A_{\alpha}$ are varied along a path in $6 \mathrm{D}$ parameter space, the vector $|k\rangle$ traces a path on the surface of a $K$-dimensional unit sphere, and the point $\left(E_{k}, I_{k}\right)$ leaves a trace in the plane of invariants.

What if two eigenvectors are accidentally degenerate $\left(E_{k}=E_{k^{\prime}}\right)$, which happens when their energy eigenvalues cross each other at some point on the path in parameter space? Generically, the eigenvalues of the second invariant are different at the point of level degeneracy $\left(I_{k} \neq I_{k^{\prime}}\right)$. We can always choose the second invariant so that this is the case. At the crossing point the orientation of the two eigenvectors is not fixed. However, that ambiguity is removed if we impose the condition that the path of every point $\left(E_{k}, I_{k}\right)$ in the plane of invariants must be continuous.

We shall see that varying $J_{\alpha}, A_{\alpha}$ along a closed path in parameter space does not guarantee that the trace of every eigenstate in the $\left(E_{k}, I_{k}\right)$ plane is also closed. It may happen, for example, that two eigenvectors transform into each other in the course of one parameter-space loop, thus leaving an open trace in the plane of invariants, which will be closed only after a second traversal of the loop. The two kinds of quantum numbers assigned to eigenstates in different regions of parameter space as discussed previously, suggest the following scenario.

(i) If the closed path in parameter space lies entirely on the integrability hypersurface, then the traces of all eigenstates in the plane of invariants will be closed. Along the loop, level crossings occur frequently, but the labeling of all eigenstates by the action quantum numbers $m_{1}, m_{2}$ remains valid on every stretch of it.

(ii) If the path in parameter space lies entirely off the integrability hypersurface, the traces of all eigenstates will again be closed but for a different reason. Level crossings are prohibited in this region. All states are labeled by the energy sorting quantum number $n$. That label is valid along the entire loop.

(iii) If the closed path in parameter space consists of a leg $A$ on and a $\operatorname{leg} B$ off the integrability hypersurface, then the conflicting assignment of quantum numbers has the consequence that some of the traces in the plane of invariants remain open. An eigenstate $|k\rangle$ may undergo one or several level crossings on leg $A$ of the path and thus end up at a different position in the energy-level sequence at the begin- 


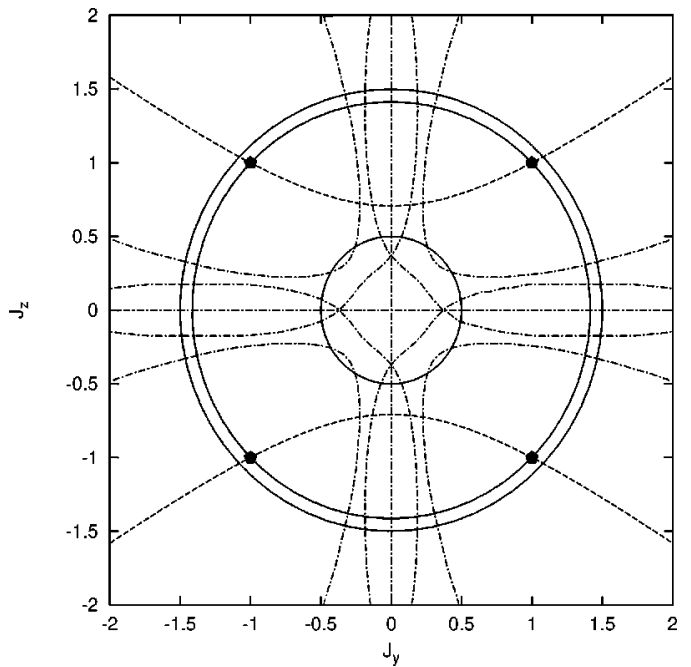

FIG. 1. Reduced parameter space $\left(J_{y}, J_{z}, A\right)$ projected onto the $\left(J_{y}, J_{z}\right)$ plane. The two dashed lines mark the intersection, $2 J_{z}^{2}$ $-J_{y}^{2}=1$, of the integrability hyperboloid with the plane $A=0$. In the integrability plane $A=0$, level degeneracies of $H_{A 1 A}^{5}$ occur along the dotted-dashed lines and multiple degeneracies at the symmetry points $\left|J_{y}\right|=\left|J_{z}\right|=1$ marked by the four pentagons. The solid circles represent projections of paths with radii $\sqrt{J_{y}^{2}+J_{z}^{2}}=\frac{1}{2}, \sqrt{2}$, $\frac{3}{2}$ along which we track the quantum invariants $E_{k}, I_{k}$.

ning of leg $B$ when the energy-sorting quantum number kicks in. As the parameters are varied along leg $B$ back to their starting values, the point $\left(E_{k}, I_{k}\right)$ is prevented from finding its way back to the original position in the plane of invariants because level crossings are now prohibited.

Not surprisingly, physical reality turns out to be more complicated. However, the observations made by this method of analysis prove to be highly illuminating in regard to the relations between symmetry, integrability, and the assignment of quantum numbers.

\section{RESULTS}

To facilitate comparison with results obtained previously, we use the same reduced 3D parameter space as in Ref. [8]. It is spanned by $J_{y}, J_{z}, A_{x}-A_{y} \equiv 2 A$ at $J_{x}=1, A_{x}+A_{y}=0, A_{z}$ $=0$ [16]. The integrability condition (2), which becomes

$$
A\left(1+J_{y}^{2}-2 J_{z}^{2}-2 A^{2}\right)=0
$$

is satisfied on a $2 \mathrm{D}$ surface consisting of the plane $A=0$ and a hyperboloid with axis at $A=0, J_{z}=0$. Embedded in this integrability surface are 1D level crossing manifolds in patterns whose complexity increases with the number of levels in the invariant (Hilbert) subspaces under consideration [8].

Individual eigenstates $|k\rangle$ will now be tracked along closed paths in this reduced parameter space. Each path selected displays distinct characteristic features in the traces on the plane of invariants $\left(E_{k}, I_{k}\right)$. Here we use $I_{k}=\langle k|\left(S_{1}^{z}\right.$ $\left.+S_{2}^{z}\right)^{2}|k\rangle$. We consider invariant (Hilbert) subspaces of symmetry class $A 1 A$ with $K=6,10$ levels corresponding to spin quantum numbers $\sigma=4,5$, respectively (see the Appendix).

Figure 1 depicts the reduced parameter space projected onto the integrability plane $A=0$. The dotted-dashed lines

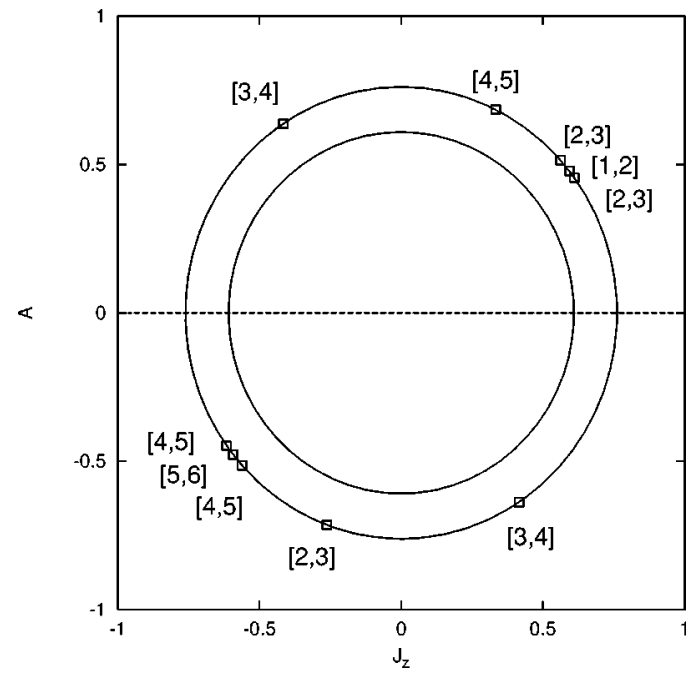

FIG. 2. Reduced parameter space $\left(J_{y}, J_{z}, A\right)$ projected onto the $\left(J_{z}, A\right)$ plane. The solid circles represent paths with $J_{z}^{2}+A^{2}$ $=0.3712,0.58$ at $J_{y}=0.4$ along which we track the quantum invariants $E_{k}, I_{k}$. The larger circle is located on the integrability hyperboloid. The positions of level crossings of $H_{A 1 A}^{4}$ states along that path are indicated by squares. The dashed line marks the integrability plane $A=0$.

represent the level crossing manifold of $H_{A 1 A}^{5}$ with $K=10$ levels in the plane $A=0$. None of the intersection points of two dotted-dashed lines involves triple or quadruple degeneracies. Each level crossing line can thus be labeled $[k, k+1]$ by the positions in the level sequence $E_{1} \leqslant E_{2}$ $\leqslant \cdots \leqslant E_{K}$ of the two levels involved in the crossing [17].

The integrability hyperboloid intersects the integrability plane along the two dashed lines. There exist $30 H_{A 1 A}^{5}$ level crossing lines on the hyperboloid. These lines intersect the plane $A=0$ at seven points on each dashed line, namely on the intersection points with dotted-dashed lines and on the symmetry points at $\left|J_{y}\right|=\left|J_{z}\right|=1$. The solid circles represent projections of paths along which we track the quantum invariants $E_{k}, I_{k}$.

A different projection of the reduced parameter space is shown in Fig. 2. The larger circle represents a path along the intersection of the integrability hyperboloid with the plane $J_{y}=0.4$. The squares on that circle mark the locations where the 10 level crossing lines on the hyperboloid for $H_{A 1 A}^{4}$ intersect the plane $J_{y}=0.4$. The smaller (concentric) circle represents a path that is located in the nonintegrable region of parameter space except for the two points where it intersects the integrability plane $A=0$ (dashed line).

\section{A. Hallmark of integrability}

The first path considered is the circle $J_{y}^{2}+J_{z}^{2}=\frac{1}{4}$ in the plane $A=0$ as shown in Fig. 1. This path does not come close to any of the symmetry points (pentagons). In Fig. 3 we have plotted the 10 levels of $H_{A 1 A}^{5}$ versus angular distance $\alpha$ on the circular path. We observe 20 pairwise crossings between six levels at the angles where the path intersects the dotted-dashed lines in Fig. 1.

No instances of level repulsion can be discerned in this plot, which is not to say that the $\alpha$ dependence of adjacent levels is uncorrelated. Take the six levels near the center of 


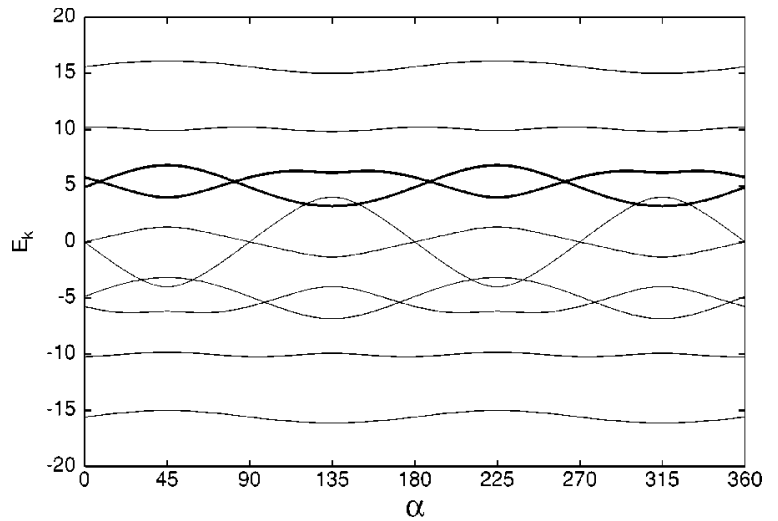

FIG. 3. Energy eigenvalues $E_{k}, k=1, \ldots, 10$ in the invariant subspace of $H_{A 1 A}^{5}$ as defined in Eq. (A1) and plotted vs the angular distance $\alpha$ on the circular path with radius $\sqrt{J_{y}^{2}+J_{z}^{2}}=0.5$ in the plane $A=0$ of the reduced parameter space $\left(J_{y}, J_{z}, A\right)$.

the spectrum. They can be divided into two groups of three levels undergoing similar oscillations along the path. The synchronicity of these oscillations is, in fact, a consequence of the (postulated) smooth dependence of the functions $H_{Q}\left(\mathcal{J}_{1}, \mathcal{J}_{2}\right)$ and $I_{Q}\left(\mathcal{J}_{1}, \mathcal{J}_{2}\right)$ on $\alpha$ for this path embedded in the integrability plane [11].

In Fig. 4 we show the traces in the $\left(E_{k}, I_{k}\right)$ plane of the two eigenstates whose levels undergo four $[7,8]$ crossings along the path (thick lines in Fig. 3). The traces are continuous, closed, and smooth. The square and the arrow indicate the starting point and the direction of the trace. Every level crossing is represented by two vertically displaced asterisks, one on each trace.

It is important to note that the traces remain perfectly smooth at the points of level crossing. The level crossings have no impact on the eigenvectors, or on the expectation values $I_{k}$. Every eigenvector loops around and returns to its original orientation in Hilbert space. Its path is largely unaffected by the presence of other eigenvectors which become instantaneously degenerate with it. It is as if vectors undergoing level crossings belonged to different invariant subspaces.

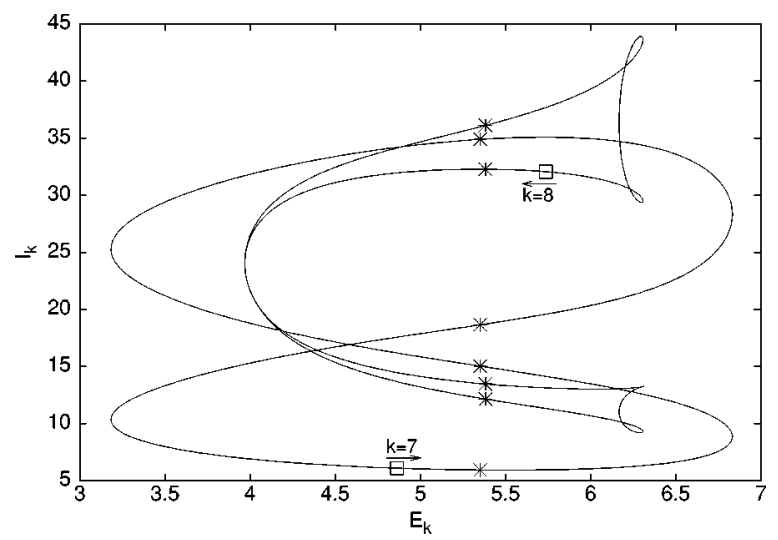

FIG. 4. Closed traces in the $\left(E_{k}, I_{k}\right)$ plane of two $H_{A 1 A}^{5}$ levels along the circular path with radius $\sqrt{J_{y}^{2}+J_{z}^{2}}=\frac{1}{2}$ in the plane $A=0$ of the reduced parameter space $\left(J_{y}, J_{z}, A\right)$. The traces start at the open squares $\left(\alpha=0^{\circ}\right)$ in the directions indicated. The asterisks mark level crossing points.

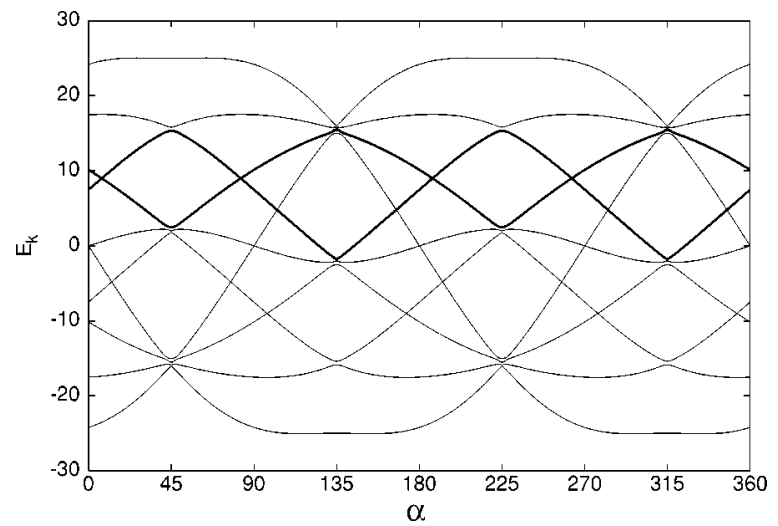

FIG. 5. Energy eigenvalues $E_{k}, k=1, \ldots, 10$ in the invariant subspace of $H_{A 1 A}^{5}$ plotted vs $\alpha$ on the path with radius $\sqrt{J_{y}^{2}+J_{z}^{2}}$ $=1.5$ in the plane $A=0$ of $\left(J_{y}, J_{z}, A\right)$ space.

The behavior of energy levels as observed in Fig. 3 and the properties of traces as seen in Fig. 4 reflect what we expect for a typical situation in an integrable system with two degrees of freedom. The two invariants $E_{k}, I_{k}$ are functions of two quantized actions $\mathcal{J}_{1}, \mathcal{J}_{2}$ with a smooth dependence on the Hamiltonian parameters. The discrete values of the actions define the natural quantum numbers of all levels, and each eigenstate maintains its identity along any path in parameter space notwithstanding the presence of level crossings. All traces produced along closed paths are therefore closed as well.

There are two sources of complication forcing on us a refinement of this description without undermining the postulated link between quantum integrability and action operators. These two complications will be discussed next before we investigate the effects of nonintegrability.

\section{B. Level repulsion near symmetry points}

The second path considered is the circle $J_{y}^{2}+J_{z}^{2}=\frac{9}{4}$ in the integrability plane $A=0$ (see Fig. 1). What makes it different from the previous path is that it passes close to the four points $\left|J_{y}\right|=\left|J_{z}\right|=1$, where additional degeneracies occur, caused by a higher symmetry.

The 10 levels of $H_{A 1 A}^{5}$ versus $\alpha$ are plotted in Fig. 5. As in Fig. 3 for the previous path, we observe 20 level crossings, each one associated with a point where the circular path intersects one of the dotted-dashed lines in Fig. 1. In addition to these crossings we observe instances of level collisions at $\alpha=n \pi / 2, n=1,3,5,7$, i.e., in the vicinity of the symmetry points.

It is instructive to compare the effects of level crossings and level collisions on the traces in the plane of invariants. In Fig. 6 we show again the trace of the point $\left(E_{k}, I_{k}\right)$ for two states that are involved in four $[7,8]$ levels crossings (thick lines in Fig. 5), now along the second path. These traces exhibit features not seen in Fig. 4.

We again observe that none of the level crossings leaves any mark on the traces, implying that the wave functions of the two eigenstates are completely unperturbed by the instantaneous level degeneracies (see asterisks). On any stretch between successive mutual crossings, both levels collide with one neighboring level, and each collision does have a dramatic effect on the traces of the states involved in the 


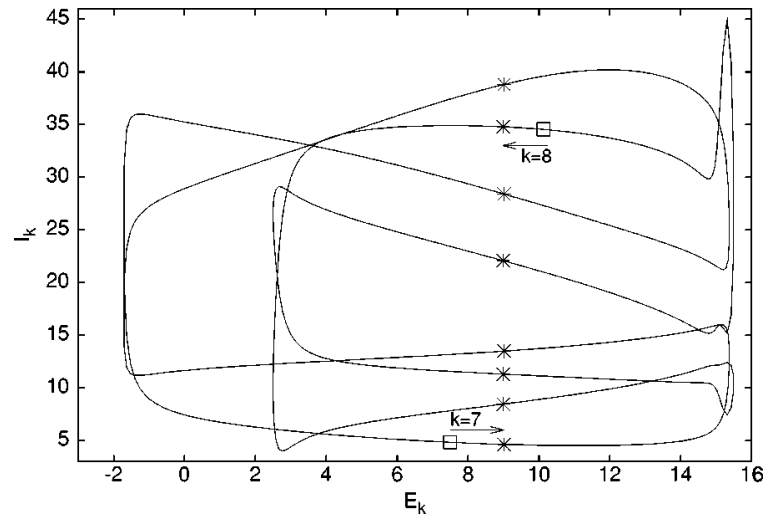

FIG. 6. Closed traces in the $\left(E_{k}, I_{k}\right)$ plane of two $H_{A 1 A}^{5}$ levels along the path with radius $\sqrt{J_{y}^{2}+J_{z}^{2}}=1.5$ in the plane $A=0$ of $\left(J_{y}, J_{z}, A\right)$ space. The traces start at the squares $\left(\alpha=0^{\circ}\right)$ in the directions indicated. The asterisks mark level crossing points.

collision. Level collisions produce precipitous changes in the second invariant $I_{k}$ near the closest encounter of the colliding levels. The rapid variation of expectation values signals a strong perturbation of the wave functions in a level collision. The presence of this characteristic signature of level collisions is as conspicuous in the traces shown in Fig. 6 as is their absence in the traces shown in Fig. 4.

In what might be called a hard level collision, the two states exchange wave functions in a manner like two billiard balls exchange momenta in a head-on collision. This makes it hard to distinguish a hard collision from a crossing in a plot such as Fig. 5 because of graphical resolution. A plot of one invariant versus the other (Fig. 6) is much more sensitive to that distinction. Here a hard level collision produces a variation in $I_{k}$ that looks almost like a discontinuity.

The phenomena observed in Figs. 5 and 6 are not in contradiction with the assertion that the invariants $E_{k}, I_{k}$ are functions of two quantum actions. It tells us, however, that the dependence of these functions on the Hamiltonian parameters is singular at the symmetry points of $H$. The phenomenon of level repulsion in the immediate vicinity of symmetry points is then caused by invariants pertaining to the higher symmetry and by the associated additional level degeneracies.

The traces of all levels depicted in Fig. 5 are closed as were all traces of the levels shown in Fig. 3. The implication is that the number of crossings between any pair of levels must be an even number. The fact is that neither the level crossings nor the level collisions can cause any confusion in the labeling of the levels by action quantum numbers along a path in the integrability plane $A=0$ as long as it avoids the points $\left|J_{y}\right|=\left|J_{z}\right|=1$ of higher symmetry with symmetry induced level degeneracies. Each eigenstate maintains its identity along such paths, or so it seems.

\section{Open traces caused by a change in symmetry}

The third path considered is the circle $J_{y}^{2}+J_{z}^{2}=2$ at $A$ $=0$ (see Fig. 1). It is embedded in the integrability plane and passes through the points $\left|J_{y}\right|=\left|J_{z}\right|=1$. The impact of these symmetry points on the energy levels is depicted in Fig. 7. What were level collisions in Fig. 5 have now turned into additional level crossings. At the symmetry points, the 10

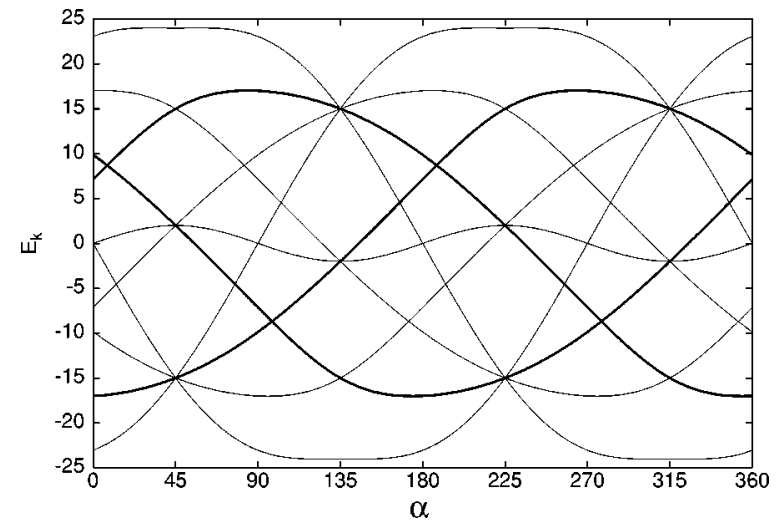

FIG. 7. Energy eigenvalues $E_{k}, k, \ldots, 10$ in the invariant subspace of $H_{A 1 A}^{5}$ plotted versus $\alpha$ on the path with radius $\sqrt{J_{y}^{2}+J_{z}^{2}}$ $=\sqrt{2}$ in the plane $A=0$ of $\left(J_{y}, J_{z}, A\right)$ space.

levels combine into a singlet, a doublet, a triplet, and a quadruplet. No instances of level repulsion are observable anymore.

The absence of level collisions along this path is confirmed by a study of the traces in the $\left(E_{k}, I_{k}\right)$ plane. In Fig. 8 we show the traces of the two states that again start in the seventh and eighth positions of the level sequence. Gone are the rapid near-vertical displacements which we have identified in Fig. 6 and which were caused by level collisions. The traces in Fig. 8 are as unaffected by the new symmetry induced level crossings as they are oblivious of crossings elsewhere in the integrability plane.

However, a striking new feature makes its appearance in Fig. 8. The traces do not close in themselves after one loop around the circular path in parameter space. The eighth level becomes the seventh level after one loop, and then turns into the second level after two loops. Only after the third loop does it end up in the original eighth position of the level sequence.

In Fig. 7 the three levels involved in that loop are drawn as thick lines. Inspection shows that there are two further groups of three states which transform into each other as the parameter values loop around the circle. That leaves one state (near the center of the spectrum) whose trace closes in itself after one loop.

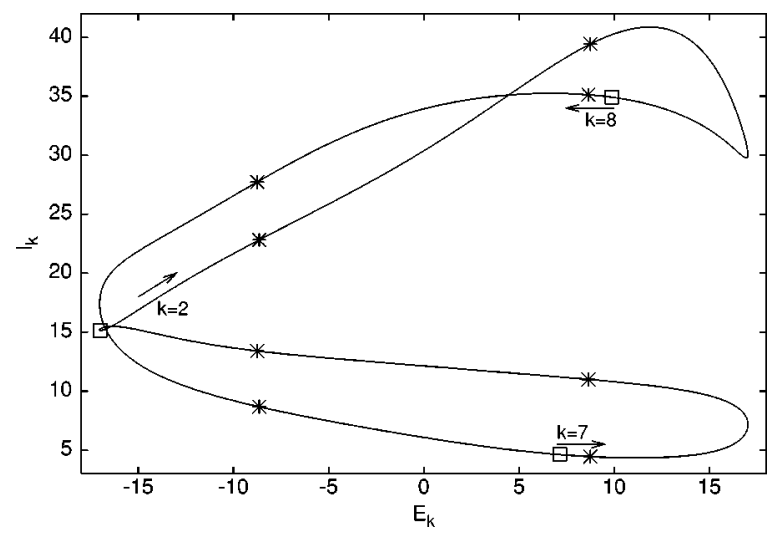

FIG. 8. Open traces in the $\left(E_{k}, I_{k}\right)$ plane of three $H_{A 1 A}^{5}$ levels along the path with radius $\sqrt{J_{y}^{2}+J_{z}^{2}}=\sqrt{2}$ in the plane $A=0$ of $\left(J_{y}, J_{z}, A\right)$ space. The traces start at the squares $\left(\alpha=0^{\circ}\right)$ in the directions indicated The asterisks mark level crossing points. 
Is this phenomenon of levels transforming into each other compatible with the notion that the invariants are functions of the quantized actions with a smooth dependence on the Hamiltonian parameters? Yes if we allow the dependence on the parameters to be singular at points of higher symmetry within the integrability manifold. The presence of such singularities was already suggested by the level collisions observed in Figs. 5 and 6. The results of Figs. 7 and 8 confirm the singular parameter dependence from a different vantage point.

When we start with the second path in parameter space (Sec. III B) and increase the radius of the circle gradually toward that of the third path, we observe a gradual hardening of the level collisions near the symmetry points. The hardening is characterized by increasingly sharp curvatures in the graphs of $E_{k}$ versus $\alpha$ (Fig. 5) and by increasingly rapid vertical variations in the graphs $I_{k}$ versus $E_{k}$ (Fig. 6).

In the limiting case of this path, the sharply curved but smooth bends in the graph $E_{k}$ versus $\alpha$ turn into cusps, and the fast but smooth vertical variations in the graphs $I_{k}$ versus $E_{k}$ turn into discontinuities. An infinitely hard level collision is indistinguishable from a level crossing. In Figs. 7 and 8 smooth segments of graphs between singularities that belong to different colliding levels are rejoined to form entirely smooth graphs of crossing levels.

Hence, if we insist that all levels maintain their identity along any closed path in the integrability plane $A=0$, we must interpret all level crossings that take place at the points of higher symmetry, $\left|J_{y}\right|=\left|J_{z}\right|=1$, as infinitely hard level collisions. All the evidence accumulated thus far still supports the existence of the functions $H_{Q}\left(\mathcal{J}_{1}, \mathcal{J}_{2}\right)$ and $I_{Q}\left(\mathcal{J}_{1}, \mathcal{J}_{2}\right)$ with a smooth parameter dependence on the integrability manifold, provided we allow for singularities at points of higher symmetry.

Before we discuss the strongly contrasting properties of quantum invariants along paths that are not fully embedded in the integrability manifold of (1), we should report on yet another feature that complicates the interpretation of the integrable cases.

\section{Open traces caused by topology}

The circle $A^{2}+J_{z}^{2}=0.58$ with center at $J_{y}=0.4$ is the fourth path along which we study the behavior of quantum invariants. This path represents a circular section of the integrability hyperboloid (3) (see Fig. 2). Like the first path considered, it does not pass near any point in parameter space where symmetry induced level degeneracies occur.

The angular dependence of the six $H_{A 1 A}^{4}$ levels, depicted in Fig. 9, does indeed not show any level collisions just as was the case in Fig. 3 for the first path. All levels undergo several crossings along this path, and none of the crossings has any noticeable effect on the quantum invariants $E_{k}, I_{k}$ plotted in Fig. 10.

Nevertheless, there is a major difference between the evolution of eigenstates along these two paths. Each one of the six levels shown in Fig. 9 transforms into a different level in the course of one loop of the path around the integrability hyperboloid. It takes three loops for every eigenstate to return to its original position in the level sequence. On the plane of invariants this phenomenon is reflected in open

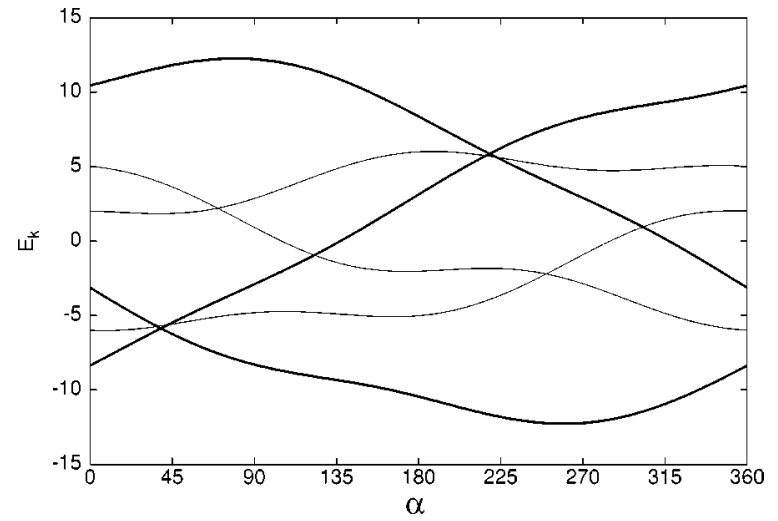

FIG. 9. Energy eigenvalues $E_{k}, k=1, \ldots, 6$ in the invariant subspace of $H_{A 1 A}^{4}$ plotted versus $\alpha$ on the path with radius $\sqrt{A^{2}+J_{z}^{2}}=\sqrt{0.58}$ at $J_{y}=0.4$ embedded in the integrability hyperboloid of $\left(J_{y}, J_{z}, A\right)$ space.

traces that connect to form two rings of three segments each as shown in Fig. 10. The two sets of levels are distinguished by line thickness.

Unlike in the previous situation (Sec. III C), here the open-trace phenomenon cannot be attributed to a change of symmetry along the path. What distinguishes the first path, where open traces do not occur from the fourth path, where they do occur, is that only the former can be shrunk to a point without leaving the integrability manifold. Hence the multiple connectedness of the integrability hyperboloid forces us to allow for functions $H_{Q}\left(\mathcal{J}_{1}, \mathcal{J}_{2}\right)$ and $I_{Q}\left(\mathcal{J}_{1}, \mathcal{J}_{2}\right)$ whose dependence on the Hamiltonian parameters is still smooth but multiple valued.

With these concessions, the signature properties of quantum integrability postulated above remain fully intact. The quantum invariants $E_{k}, I_{k}$ exhibit strongly contrasting features when observed along paths that are not embedded in the integrability manifold. Visualizing these differences does not depend on a statistical analysis. They are unmistakenly identifiable in systems with very few levels.

\section{E. Level repulsion due to nonintegrability}

For a direct comparison with the previous situation, we now choose a circle with the same center as the fourth path

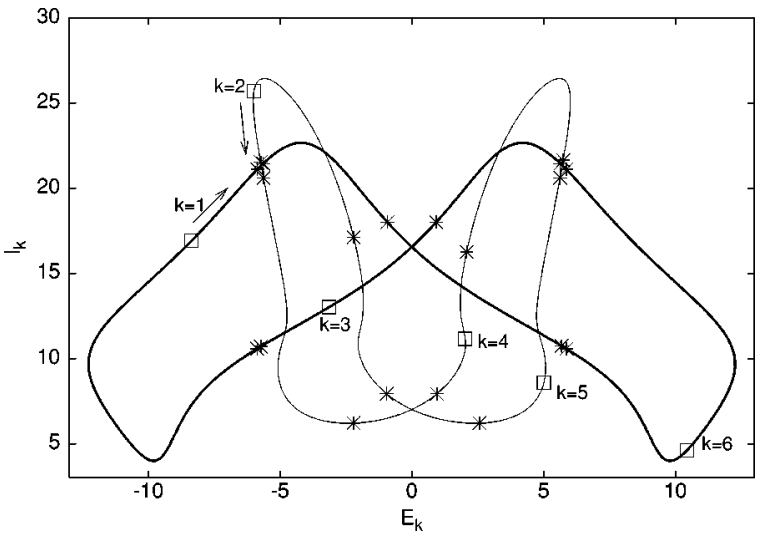

FIG. 10. Open traces in the $\left(E_{k}, I_{k}\right)$ plane of all six $H_{A 1 A}^{4}$ levels along the path with radius $\sqrt{A^{2}+J_{z}^{2}}=\sqrt{0.58}$ at $J_{y}=0.4$ on the integrability hyperboloid in $\left(J_{y}, J_{z}, A\right)$ space. The traces start at the squares $\left(\alpha=0^{\circ}\right)$ in the directions indicated. The asterisks mark level crossing points. 


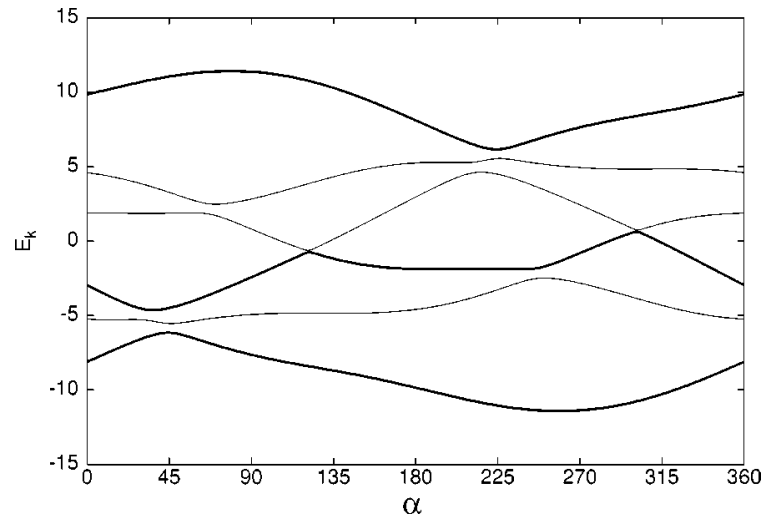

FIG. 11. Energy eigenvalues $E_{k}, k=1, \ldots, 6$ in the invariant subspace of $H_{A 1 A}^{4}$ plotted vs $\alpha$ on the path with radius $\sqrt{A^{2}+J_{z}^{2}}$ $=\sqrt{0.3712}$ at $J_{y}=0.4$ off the integrability hyperboloid in $\left(J_{y}, J_{z}, A\right)$ space.

and a somewhat smaller radius, $J_{z}^{2}+A^{2}=0.3712$. This fifth path lies off the integrability manifold except for two points where it intersects the integrability plane $A=0$ (see Fig. 2). However, no level degeneracies occur at these intersection points.

The six $H_{A 1 A}^{4}$ levels versus $\alpha$ along the fifth path are plotted in Fig. 11. Even though the resulting pattern is vaguely similar to that observed in Fig. 9, the differences are clear cut. All level crossings have turned into level collisions.

Most of the collisions are fairly soft. The two hardest collisions are barely resolved as such on the scale of Fig. 12. None of the levels transform into each other any more. The levels are now naturally labeled by the energy sorting quantum number. Each open segment of the traces shown in Fig. 10 has turned into a closed trace. All level collisions, especially the hard ones, leave the characteristic marks on the traces in the form of a rapidly varying second invariant $I_{k}$.

If we were to move the fifth path closer to the integrability hyperboloid by increasing its radius (see Fig. 2), we could observe a gradual hardening of all level collisions. The level configurations as shown in Fig. 11 would increasingly resemble those in Fig. 9. The traces as shown in Fig. 12, how-

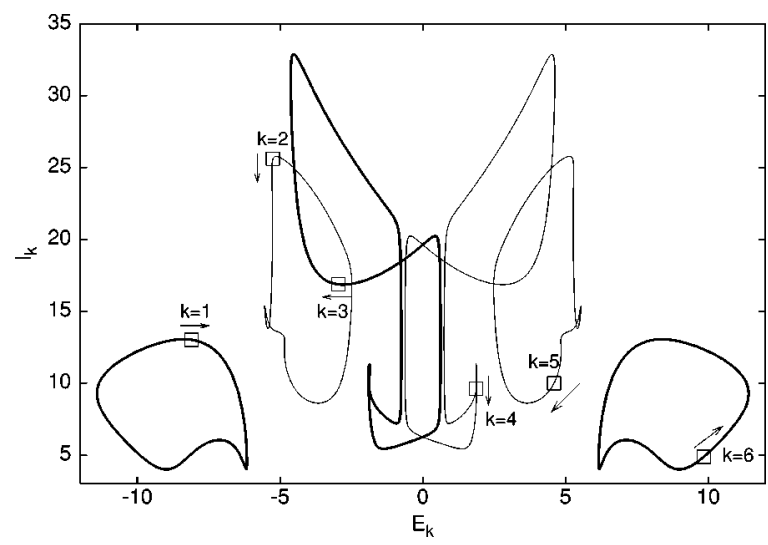

FIG. 12. Closed traces in the $\left(E_{k}, I_{k}\right)$ plane of all six $H_{A 1 A}^{4}$ levels along the path with radius $\sqrt{A^{2}+J_{z}^{2}}=\sqrt{0.3712}$ at $J_{y}=0.4$ off the integrability hyperboloid in $\left(J_{y}, J_{z}, A\right)$ space. The traces start at the the squares $\left(\alpha=0^{\circ}\right)$ in the directions indicated. ever, would remain very different from those pertaining to the integrable case (Fig. 10).

Only in the limiting case where the fifth path merges with the fourth path would the closed traces of the nonintegrable model break into segments connected by vertical lines. The ends of each segment would then rejoin ends of other segments to form the smooth rings of open traces shown in Fig. 10.

Similar observations are made upon lifting the first path off the integrability plane $A=0$ to a plane at $A \neq 0$. All the level crossings that exist in Fig. 3, for example, turn into level collisions. The closed traces such as those shown in Fig. 4 break into pieces whose ends rejoin via near vertical lines into a new set of closed traces.

Along the second path we had observed (in Fig. 5) level crossings (due to integrability) and level collisions (due to nearby points of higher symmetry). Lifting this path off the integrability plane again removes all level crossings and results in a set of closed traces. The characteristic marks of level collisions on the traces in the $\left(E_{k}, I_{k}\right)$ plane are the same no matter whether they are caused by a reduced symmetry or by nonintegrability.

Lifting the third path off the integrability plane has the same effects on the level crossings attributed to integrability and the level crossings attributed to the higher symmetry at selected points in parameter space (Fig. 7). All are removed indiscriminately.

\section{F. Open traces caused by nonintegrability}

The conflicting assignments of quantum numbers to eigenstates for parameter values on and off the integrability manifold is most compellingly documented when we pick a path in parameter space that is only partially embedded in the integrability manifold.

The sixth path considered in this study of quantum invariants is a modification of the first path (Sec. III A) with the same projection in Fig. 1. Whereas the first path was embedded in the integrability plane $A=0$, the sixth path has a variable height relative to that plane: $A(\alpha)=0.3 \cos ^{2}(\alpha / 2)$. It touches down to the integrability plane at a single point $(\alpha$ $=180^{\circ}$ ), where a $[5,6]$ level crossing takes place.

Along this path there exist no other level crossings. All the other crossings that existed in Fig. 3 for the first path are now replaced by level collisions (see Fig. 13).

The inevitable consequence of having a single level crossing along a closed path in parameter space is the existence of a pair of open traces in the plane of invariants, namely the traces of the states that undergo the $[5,6]$ crossing at $\alpha$ $=180^{\circ}$. These traces are shown in Fig. 14. The ends of the solid and dashed lines form a single loop, which is traced in the direction indicated.

What causes here an open trace in the plane of invariants is obviously akin to what had caused an open trace in the situation described in Sec. III C. In both cases two levels cross once due to particular circumstances at one point of the path, and are thus prevented from crossing back to their original position in the level sequence on the remaining stretch of the path. In Sec. IIIC the particular circumstance was a higher symmetry, here it is integrability. 


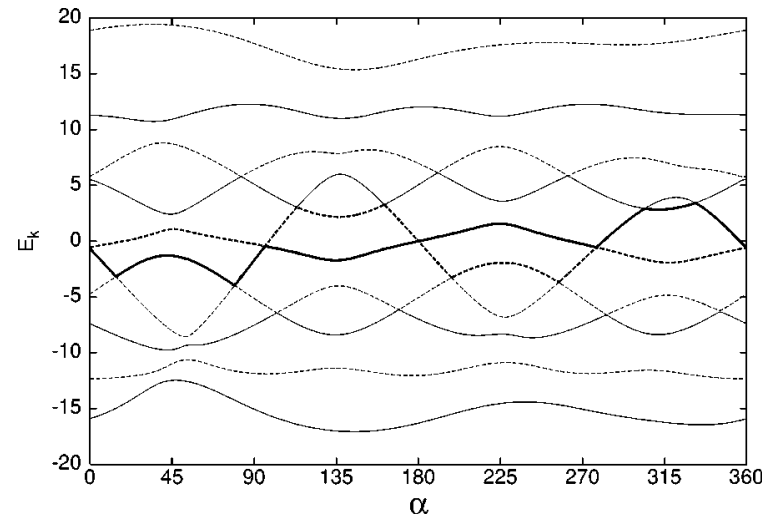

FIG. 13. Energy eigenvalues $E_{k}, k=1, \ldots, 10$ in the invariant subspace of $H_{A 1 A}^{5}$ plotted vs $\alpha$ on the path with radius $\sqrt{J_{y}^{2}+J_{z}^{2}}$ $=0.5$ at $A=0.3 \cos ^{2}(\alpha / 2)$ in $\left(J_{y}, J_{z}, A\right)$ space.

\section{INTERPRETATION}

The study of quantum invariants along closed paths through parameter space indicates that a change in symmetry and a change in integrability status produce related phenomena. In some dynamical systems, the conservation laws that guarantee integrability are direct consequences (via Noether's theorem) of continuous symmetries. Switching from integrability to nonintegrability is then accompanied by a reduction in symmetry.

In the two-spin model (1), the presence of a (continuous rotational) $O(2)$ or higher symmetry in spin space does indeed imply the existence of a second integral of the motion, namely the component of the total spin along the symmetry axis, and integrability is guaranteed. However, a second integral of the motion was shown to exist for certain parameter values even in the absence of a continuous rotational symmetry [9]. Does integrability in that case indicate the presence of a hidden symmetry?

Classical integrability guarantees that the Hamiltonian (1) can be expressed as a function of the two action variables: $H=H_{C}\left(\mathcal{J}_{1}, \mathcal{J}_{2}\right)$. The cyclical nature of the angle coordinates thus implies that $H_{C}$ is invariant with respect to continuous rotationlike transformations in phase space. Since this is not

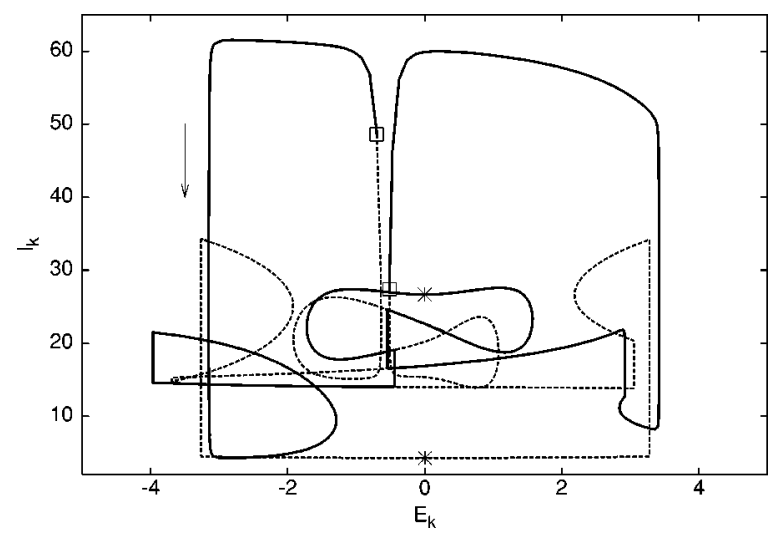

FIG. 14. Open traces in the $\left(E_{k}, I_{k}\right)$ plane of the two $H_{A 1 A}^{5}$ levels which undergoes one $[5,6]$ crossings along the path with radius $\sqrt{J_{y}^{2}+J_{z}^{2}}=0.5$ at $A=0.3 \cos ^{2}(\alpha / 2)$ in $\left(J_{y}, J_{z}, A\right)$ space. The traces start at the squares in the direction indicated. The asterisk on each trace marks the level crossing point at $\alpha=180^{\circ}$.
TABLE I. Symmetry-adapted basis vectors for integer $\sigma$. The local spin quantum numbers satisfy the relations $0<l_{1} \leqslant \sigma,-l_{1}$ $<l_{2}<l_{1}$. The subspace dimensionality $K$ is $\frac{1}{2}(\sigma+1)(\sigma+2)$ for $A 1 S, \frac{1}{2} \sigma(\sigma-1)$ for $A 1 A$, and $\frac{1}{2} \sigma(\sigma+1)$ for the other six classes.

\begin{tabular}{|c|c|}
\hline$A 1 S$ & $\begin{array}{c}|00\rangle,\left(\left|l_{1}, l_{1}\right\rangle+\left|-l_{1},-l_{1}\right\rangle\right) / \sqrt{2}, \\
\left(\left|l_{1},-l_{1}\right\rangle+\left|-l_{1}, l_{1}\right\rangle\right) / \sqrt{2} \\
\left(\left|l_{1}, l_{2}\right\rangle+\left|-l_{1},-l_{2}\right\rangle+\left|l_{2}, l_{1}\right\rangle+\left|-l_{2},-l_{1}\right\rangle\right) / 2 \\
l_{1}+l_{2} \text { even }\end{array}$ \\
\hline$A 1 A$ & $\begin{array}{c}\left(\left|l_{1}, l_{2}\right\rangle+\left|-l_{1},-l_{2}\right\rangle-\left|l_{2}, l_{1}\right\rangle-\left|-l_{2},-l_{1}\right\rangle\right) / 2 \\
l_{1}+l_{2} \text { even }\end{array}$ \\
\hline$B 1 S$ & $\begin{array}{c}\left(\left|l_{1}, l_{1}\right\rangle-\left|-l_{1},-l_{1}\right\rangle\right) / \sqrt{2} \\
\left(\left|l_{1}, l_{2}\right\rangle-\left|-l_{1},-l_{2}\right\rangle+\left|l_{2}, l_{1}\right\rangle-\left|-l_{2},-l_{1}\right\rangle\right) / 2 \\
l_{1}+l_{2} \text { even }\end{array}$ \\
\hline$B 1 A$ & $\begin{array}{c}\left(\left|l_{1},-l_{1}\right\rangle-\left|-l_{1}, l_{1}\right\rangle\right) / \sqrt{2} \\
\left(\left|l_{1}, l_{2}\right\rangle-\left|-l_{1},-l_{2}\right\rangle-\left|l_{2}, l_{1}\right\rangle+\left|-l_{2},-l_{1}\right\rangle\right) / 2 \\
l_{1}+l_{2} \text { even }\end{array}$ \\
\hline$B 2 S$ & $\begin{array}{c}\left(\left|l_{1}, l_{2}\right\rangle-\left|-l_{1},-l_{2}\right\rangle+\left|l_{2}, l_{1}\right\rangle-\left|-l_{2},-l_{1}\right\rangle\right) / 2 \\
l_{1}+l_{2} \text { odd }\end{array}$ \\
\hline$B 2 A$ & $\begin{array}{c}\left(\left|l_{1}, l_{2}\right\rangle-\left|-l_{1},-l_{2}\right\rangle-\left|l_{2}, l_{1}\right\rangle+\left|-l_{2},-l_{1}\right\rangle\right) / 2 \\
l_{1}+l_{2} \text { odd }\end{array}$ \\
\hline$B 3 S$ & $\begin{array}{c}\left(\left|l_{1}, l_{2}\right\rangle+\left|-l_{1},-l_{2}\right\rangle+\left|l_{2}, l_{1}\right\rangle+\left|-l_{2},-l_{1}\right\rangle\right) / 2 \\
l_{1}+l_{2} \text { odd }\end{array}$ \\
\hline$B 3 A$ & $\begin{array}{c}\left(\left|l_{1}, l_{2}\right\rangle+\left|-l_{1},-l_{2}\right\rangle-\left|l_{2}, l_{1}\right\rangle-\left|-l_{2},-l_{1}\right\rangle\right) / 2 \\
l_{1}+l_{2} \text { odd }\end{array}$ \\
\hline
\end{tabular}

related to a continuous symmetry in configuration space, it is appropriate to call it a hidden symmetry.

For a description of the impact of symmetries on the level spectrum of the quantum two-spin model, it is useful to distinguish three kinds of symmetry: discrete symmetries, continuous symmetries, and hidden symmetries.

Discrete symmetries have no bearing on the classical integrability property, but they do affect the shapes of phasespace trajectories. Quantum mechanically, they divide the Hilbert space of $H$ into invariant subspaces. In general, this does not result in symmetry-induced level degeneracies, but it does lead to accidental degeneracies between levels belonging to different invariant subspaces. Such level crossings exist independently of whether or not $H$ is integrable.

Hidden symmetries, which guarantee classical integrability, cause additional accidental level degeneracies, namely between states within one of the invariant subspaces pertaining to any existing discrete symmetry.

Continuous symmetries, in essence, combine the effects of the discrete and hidden symmetries, and allow accidental intersubspace degeneracies. In addition to these effects, continuous symmetries (sometimes in tandem with discrete symmetries) produce level degeneracies of a permanent nature, the so-called symmetry-induced level degeneracies.

There exists a hierarchy of symmetries in the two-spin model (1): (S0) In the absence of any symmetry, there are no level degeneracies. All levels will collide when Hamiltonian parameters are varied. This situation can be realized by an external magnetic field. (S1) The existence of discrete symmetries alone produces finite- $D$ invariant Hilbert subspaces. 
Level crossings exist between states belonging to different subspaces. Levels within any subspace collide. (S2) The existence of hidden symmetries in addition to discrete symmetries produces level crossings between states in the same invariant subspace. (S3) The continuous symmetries produce permanent degeneracies in certain regions of parameter space.

There exists a hierarchy of level collisions which corresponds to the hierarchy of symmetries. (S1) $\rightarrow(\mathrm{S} 0)$ Intersubspace level crossings in the presence of discrete symmetries turn into level collisions when discrete symmetries are removed. (S2) $\rightarrow$ (S1) Intrasubspace level crossings turn into level collisions when the hidden symmetries are removed, i.e., when the integrability is destroyed. $(\mathrm{S} 3) \rightarrow(\mathrm{S} 2)$ Symmetry-induced level degeneracies associated with a continuous symmetry are removed outside the range of that symmetry irrespective of the presence or absence of the hidden symmetry.

Some level crossings along paths through symmetry points in parameter space turn into level collisions along nearby paths that miss the symmetry point. Other level crossings are insensitive to whether the path hits or misses the symmetry point. They are the product of the hidden symmetry.

All phenomena observed in the quantum invariants $E_{k}, I_{k}$ along closed paths on, off, and across the integrability manifold, indicate that the effects of a change in integrability status are akin to the effects of a change in symmetry. All observations point to the existence of a hidden symmetry that accompanies quantum integrability.

In the classical limit, this hidden symmetry manifests itself in phase space when viewed from a particular coordinate system - the action-angle coordinates. The same hidden symmetry must also exist in the quantum system, but only on the integrability manifold. Even though nonintegrability is not to be taken literally in the quantum case, the presence or absence of that hidden symmetry has consequences that are equally clear-cut as in the classical limit.

\section{ACKNOWLEDGMENTS}

This work was supported by the Research Office of the University of Rhode Island. We are very grateful to Joachim Stolze for his comments and suggestions relating to this work.

\section{APPENDIX: DISCRETE SYMMETRIES}

The (discrete) symmetry group relevant for the general 2-spin Hamiltonian (1) is $D_{2} \otimes S_{2}$, where $D_{2}$ contains the three twofold rotations $C_{2}^{\alpha}, \alpha=x, y, z$ about the coordinate
TABLE II. Symmetry-adapted basis vectors for half-integer $\sigma$. The local spin quantum numbers satisfy the relations $0<l_{1} \leqslant \sigma$, $-l_{1}<l_{2}<l_{1}$. The subspace dimensionality $K$ is $\frac{1}{8}\left(4 \sigma^{2}-1\right)$ for symmetric and $\frac{1}{8}(2 \sigma+1)(2 \sigma+3)$ for antisymmetric representations.

\begin{tabular}{|c|c|}
\hline$A 1 S$ & $\begin{array}{c}\left(\left|l_{1}, l_{2}\right\rangle-\left|-l_{1},-l_{2}\right\rangle+\left|l_{2}, l_{1}\right\rangle-\left|-l_{2},-l_{1}\right\rangle\right) / 2 \\
l_{1}+l_{2} \text { even }\end{array}$ \\
\hline$A 1 A$ & $\begin{array}{c}\left(\left|l_{1},-l_{1}\right\rangle-\left|-l_{1}, l_{1}\right\rangle\right) / \sqrt{2} \\
\left(\left|l_{1}, l_{2}\right\rangle-\left|-l_{1},-l_{2}\right\rangle-\left|l_{2}, l_{1}\right\rangle+\left|-l_{2},-l_{1}\right\rangle\right) / 2 \\
l_{1}+l_{2} \text { even }\end{array}$ \\
\hline$B 1 S$ & $\begin{array}{c}\left(\left|l_{1},-l_{1}\right\rangle+\left|-l_{1}, l_{1}\right\rangle\right) / \sqrt{2} \\
\left(\left|l_{1}, l_{2}\right\rangle+\left|-l_{1},-l_{2}\right\rangle+\left|l_{2}, l_{1}\right\rangle+\left|-l_{2},-l_{1}\right\rangle\right) / 2 \\
l_{1}+l_{2} \text { even }\end{array}$ \\
\hline$B 1 A$ & $\begin{array}{c}\left(\left|l_{1}, l_{2}\right\rangle+\left|-l_{1},-l_{2}\right\rangle-\left|l_{2}, l_{1}\right\rangle-\left|-l_{2},-l_{1}\right\rangle\right) / 2 \\
l_{1}+l_{2} \text { even }\end{array}$ \\
\hline$B 2 S$ & $\begin{array}{c}\left(\left|l_{1}, l_{1}\right\rangle+\left|-l_{1},-l_{1}\right\rangle\right) / \sqrt{2} \\
\left(\left|l_{1}, l_{2}\right\rangle+\left|-l_{1},-l_{2}\right\rangle+\left|l_{2}, l_{1}\right\rangle+\left|-l_{2},-l_{1}\right\rangle\right) / 2 \\
l_{1}+l_{2} \text { odd }\end{array}$ \\
\hline$B 2 A$ & $\begin{array}{c}\left(\left|l_{1}, l_{2}\right\rangle+\left|-l_{1},-l_{2}\right\rangle-\left|l_{2}, l_{1}\right\rangle-\left|-l_{2},-l_{1}\right\rangle\right) / 2 \\
l_{1}+l_{2} \text { odd }\end{array}$ \\
\hline$B 3 S$ & $\begin{array}{c}\left(\left|l_{1}, l_{1}\right\rangle-\left|-l_{1},-l_{1}\right\rangle\right) / \sqrt{2} \\
\left(\left|l_{1}, l_{2}\right\rangle-\left|-l_{1},-l_{2}\right\rangle+\left|l_{2}, l_{1}\right\rangle-\left|-l_{2},-l_{1}\right\rangle\right) / 2 \\
l_{1}+l_{2} \text { odd }\end{array}$ \\
\hline$B 3 A$ & $\begin{array}{c}\left(\left|l_{1}, l_{2}\right\rangle-\left|-l_{1},-l_{2}\right\rangle-\left|l_{2}, l_{1}\right\rangle+\left|-l_{2},-l_{1}\right\rangle\right) / 2 \\
l_{1}+l_{2} \text { odd }\end{array}$ \\
\hline
\end{tabular}

axes, and $S_{2}$ the permutations of the two spins. The eight irreducible representations of $D_{2} \otimes S_{2}$ are named $A 1 S, A 1 A, B 1 S, B 1 A, B 2 S, B 2 A, B 3 S, B 3 A$, where $S(A)$ stand for (anti-)symmetric under permutation and $A 1, B 1, B 2, B 3 \quad$ for $\quad\left(C_{2}^{x}, C_{2}^{y}, C_{2}^{z}\right)=(1,1,1),(1,-1,-1)$, $(-1,1,-1),(-1,-1,1)$, respectively $[4,18]$.

The basis vectors with transformation properties corresponding to the eight different irreducible representations $R$ are listed in Table I for integer $\sigma$ and in Table II for halfinteger $\sigma$. The Hamiltonian matrix can then be expressed in the form

$$
\begin{gathered}
H=\underset{R, \sigma}{\oplus} H_{R}^{\sigma} \\
\end{gathered}
$$

with blocks of dimensionalities $K=1,3,6,10, \ldots$ in 16 different realizations, two for each symmetry class (one with integer $\sigma$ and one with half-integer $\sigma$ ) [19].
[1] S. Weigert, Physica D 56, 107 (1992).

[2] S. Weigert and G. Müller, Chaos, Solitons and Fractals 5, 1419 (1995)

[3] A. Peres, Phys. Rev. Lett. 53, 1711 (1984).

[4] N. Srivastava and G. Müller, Z. Phys. B 81, 137 (1990).

[5] M. C. Gutzwiller, Chaos in Classical and Quantum Mechanics (Springer-Verlag, New York, 1990).
[6] L. E. Reichl, The Transition to Chaos in Conservative Classical Systems: Quantum Manifestations (Springer-Verlag, New York, 1992).

[7] M. C. Gutzwiller, Am. J. Phys. 66, 304 (1998).

[8] V. V. Stepanov and G. Müller, Phys. Rev. E 58, 5720 (1998).

[9] E. Magyari, H. Thomas, R. Weber, C. Kaufman, and G. Müller, Z. Phys. B 65, 363 (1987). 
[10] Only one exception to that rule is known. The energy level $E=0$ is also degenerate on a $3 \mathrm{D}$ manifold away from the integrability hypersurface (2) (see Refs. [8] and [18]).

[11] V. V. Stepanov and G. Müller (unpublished).

[12] S. D. Augustin and H. Rabitz, J. Chem. Phys. 71, 4956 (1979).

[13] R. G. Newton, Ann. Phys. (N.Y.) 124, 327 (1980).

[14] A. Luis and L. L. Sánchez-Soto, Phys. Rev. A 48, 752 (1993).

[15] A natural choice of $A$ would be an operator that commutes with $H$ on the integrability hypersurface as can be constructed from the known second classical integral of the motion (Ref. [9]), but any polynomial of the $S_{i}^{\alpha}$ will produce expectation values with equivalent properties (Refs. [3] and [9]). Only the diagonal matrix elements matter in this context.

[16] The $J_{\alpha}, A_{\alpha}$ are measured in arbitrary energy units divided by $\hbar^{2}$. To avoid cluttered notations in the text and in the figures, this unit will be suppressed in all explicit results that follow.

[17] The levels involved in the crossings are $[k, k+1]$ with $k$ $=5,7,4,4,7,5,3,6,6,3,5$ starting from either end of the horizontal line counterclockwise along the frame of the graph.

[18] R. Weber, doctoral dissertation, University of Basel, 1988.

[19] $K=1$ has only 15 realizations. 